How to reference this article Megale, T. (2019). II professionismo delle attrici: stato degli studi e nuove domande. Italica Wratislaviensia, 10(2), 15-36.

DOI: http://dx.doi.org/10.15804/IW.2019.10.1.14

\title{
Saggio introduttivo
}

\author{
Teresa Megale \\ Università degli Studi di Firenze \\ teresa.megale@unifi.it \\ ORCID: 0000-0003-1142-9948
}

\section{IL PROFESSIONISMO DELLE ATTRICI: STATO DEGLI STUDI E NUOVE DOMANDE}

\author{
BEING A PROFESSIONAL ACTRESS. \\ AN OVERVIEW OF THE EXISTING BODY \\ OF RESEARCH AND NEW INQUIRES TO BE MADE
}

\begin{abstract}
In the second half of the $16^{\text {th }}$ century, the arrival of women on the stage caused a revolution, the effects of which extended to the entirety of modern society. A troupe on the move and always at risk, faced with waves of condemnation, the women actors, nomads by trade, were preceded by a diffidence that was both twofold and simultaneous: towards the principal makings of professional work in the theatrical arts and towards the moral qualities of which they had been regarded as the heralds, not individually but as a social group. The process of deconstructing the artistic identity of these pioneering actresses, who tended to be equated with harlots or to their opposites, that is, virgin actresses, was not limited to the first appearance of women on the professional scene, but, rather, it became a long-standing historical category. The article is concerned with removing prior moral judgments that were stratified over the actresses and freeing the historiographic field from preconceived viewpoints; it is concerned with dismantling misogyny and anachronistic sidelong glances. Between masks and prejudices regarding the perturbing aesthetic perception represented by the entrance of women into the world of art, distinguishing that which separates the actress's profession from sexual adventures, talent from reputation, art from seduction, is essential to provide new historiographic categories and new coordinates to the study of the commedia dell'arte. In this way, other past experiences come to light: the launch towards professionalism, the role of marriage, and the violence that women underwent are a few of the problematic issues to reconsider and overcome based on prior documentary studies.
\end{abstract}

Keywords: female professionalism, female leaders of theatre companies, women's dramaturgy, commedia dell'arte, theatre historiography 
Tntorno alla seconda metà del XVI secolo, l'apparizione sulla scena delle attrici assunse i contorni marcati di una rivoluzione, che da teatrale presto si mutò in culturale e sociale. In modo sensibile il professionismo attorico delle donne italiane incise sulla mentalità collettiva, scosse dalle fondamenta la cultura dominante dell'assolutismo ed esercitò una forza d'urto di considerevole portata storica. Perciò è opportuno riconsiderare i confini del fenomeno storico alla luce degli avanzamenti delle ricerche e degli studi intorno a quel complesso mondo artistico universalmente noto come Commedia dell'Arte.

L'apparire delle donne in scena sin verso la metà del Cinquecento con Vincenza Armani e nell'emblematico sesto decennio del secolo con almeno due attestazioni inequivoche (nell'estate del 1562 compare la romana Barbara Flaminia alla corte di Mantova ${ }^{1}$, nell'autunno del 1564, da un contratto notarile rogato a Roma il 10 ottobre si affaccia sulla scena della storia «domina Lucretia Senensis») scatenò effetti differenti e profondi, che qui si tenterà di riassumere. Sul piano filosofico mise definitivamente in crisi la certezza, salda da secoli, circa la presunta inferiorità delle donne rispetto agli uomini e la loro supposta, pregiudiziale mancanza dell'anima; sul piano sociale impresse uno sconvolgimento, mai verificatosi fino ad allora, legato al profilarsi della retribuzione di donne artiste inserite in un sistema di lavoro collettivo e comunitario; sul piano estetico impose il rinnovamento radicale del medium teatro e il suo totale riposizionamento nel sistema delle arti, mutando, una volta per tutte, il senso della vista degli spettatori con l'invenzione della percezione moderna del pubblico; sul piano squisitamente teatrale favorì molto probabilmente il passaggio dalle commedie recitate all'improvviso a quelle premeditate e consentì l'allargamento dell'offerta drammaturgica dai lazzi comici, tenuti genialmente insieme dagli Zanni, animatori di esili canovacci, al repertorio premeditato delle tragedie, tragicommedie, favole pastorali. Per tali, sommarie ragioni, la rivoluzione teatrale rappresentata dalle donne di scena, apparse sul suolo italiano e precocemente diffusesi nel resto d'Europa, fu pari, per

${ }^{1}$ Per una ridefinizione del profilo dell'attrice si veda adesso Mattioda, 2014 e Simoncini, 2016. 
impatto e peso, a quella copernicana. Il De revolutionibus orbium coelestium del grande astronomo polacco, base della scienza moderna, si può anche leggere come anticipatore del clima di effervescenza scientifica che suscitò e che per vie implicite favorì la nascita del professionismo a teatro: l'eliocentrismo copernicano, messo in luce nel trattato uscito a stampa nel 1543, idealmente precede e prepara l'arrivo del corpo sessuato femminile al centro della scena pubblica come nuovo perno intorno al quale ripensare e riordinare le componenti dello spettacolo, sul quale calamitare lo sguardo del pubblico e una inedita cultura della visione.

L'antico medium del teatro, fino ad allora sopravvissuto a scarti e sussulti storici di vario genere ed entità, in epoca moderna cambiò radicalmente volto quando le attrici italiane soppiantarono il teatro-centrismo dell'attore, cui competevano di diritto (il diritto acquisito tramite la secolare e assolutistica pratica di palcoscenico), e indistintamente, tutte le parti in commedia. Il teatro-centrismo delle attrici fu, dunque, uno dei segreti rivelati delle compagnie dell'Arte italiane. Pertanto, non suoni tendenzioso affermare che la sostituzione del travestitismo maschile con le donne in carne ed ossa abbia avuto effetti sconvolgenti, in tutto simili a quelli che suscitò la dimostrazione del sole in posizione centrale rispetto al movimento della terra e degli altri pianeti. Si è scritto e ripetuto fors'anche meccanicamente come il corpo sessuato femminile provocasse profondo turbamento: le attrici vive, non il loro simulacro fatto di trucchi e maschere, liquidarono l'indiscussa visione tolemaica del teatro nel liberare il desiderio del pubblico maschile, mentre indicavano a quello femminile le infinite possibilità espressive del corpo e della parola. Ma le donne attrici non si attardarono a scatenare solo i sensi degli astanti e a indicare nuovi orizzonti di attesa nel pubblico, composto da spettatori liberi e paganti: esse furono la prova vivente, molto pericolosa per il pensiero dominante, che il teatro è relazione con l'altro da sé e potente strumento di conoscenza di sé stessi attraverso lo sguardo degli altri.

Punto di non ritorno, l'arrivo dell'attrice sul palcoscenico, al pari della luce catturata sulle tele dei pittori caravaggeschi, lasciava gemmare risvegli di coscienza fin lì inediti e tingeva di sano naturalismo la 
sostanza del teatro. Si trattava di squarci scenici che per la loro stessa potenza visiva condussero presto l'attrice nella zona d'ombra del peccato, e di quello rubricabile come mortale: per la cultura ufficiale espressa dagli uomini di Chiesa, la donna di scena ridiventava biblicamente l'origine di ogni male, una novella Eva che ingenerava rapimenti, che stimolava impulsi, che attivava i sensi e veicolava il male tramite gli occhi e le orecchie. Exempla riprovevoli, esse spingevano «ad amore disonesto, et molto più il vederle et il vederle et udirle insieme», come coglieva l'acuto Juan de Pineda nel 1599 (Taviani, 1969, p. 121)². Ovunque, nelle pagine dei detrattori, risuonerà la drammatizzazione di contestazioni identiche, quasi un allucinato teatro delle parole sacre eretto contro il vitale teatro dei corpi profani, tramite il quale ottenere la persecuzione e la violenta compressione della nuova "invenzione" chiamata Commedia dell'Arte. Valga, a tal proposito e a scopo esemplificativo, la catena di domande retoriche inanellate dal gesuita Pietro Gambacorta nel 1585, per mezzo delle quali egli metteva a fuoco lo sfruttamento della sensualità del corpo femminile in scena, nell'analizzare - non per caso - la Serva e l'Innamorata, le due parti muliebri essenziali e fisse impegnate nel moderno gioco scenico:

il Zanni con la serva è la salsa del diavolo. Che sarà poi udire la donna parlare? E d'amore? E con l'innamorato? E scoprirsi l'un l'altro gli effetti? E trattare il modo di ritrovarsi? Che sarà vedere che l'adultero chiede un bacio e l'ottiene? Che sarà che la donna, fingendosi pazza, comparisce mezzo spogliata, o con veste trasparente in presenza d'uomini e di donne? (Taviani, 1969 , p. XCI)

Gli interrogativi sono eloquenti dello sconcerto e del turbamento generale causati dall'apparire della donna attrice e della sua capacità di ricorrere a registri recitativi inclini all'eccesso, che paiono attinti di-

2 Sul diffuso concetto di peccato legato alle donne di scena, si legga - ad esempio la deliberazione del Senato genovese dell'agosto 1584: «Essendosi seguiti parechi disordini con le donne delle Commedie il Serenissimo Senato ordinò fossero mandate fuor della città. $\mathrm{E}$ così fu fatto, e tanto più si è confirmato questo che tutti i teologhi affermarono che sia peccato mortale recitando cose per quelli le sentano» (Inventione di Giulio Pallavicino, 1975, p. 54). 
rettamente dal lessico di antiche menadi. Affogato nel mare umanistico dell'apollineo teatro erudito, il dionisiaco sembrava riemergere e riacquistare vigore sulle scene del teatro professionistico e riottenere le migliori incarnazioni nella metamorfica arte della finzione femminile.

L'avvento delle professioniste è, dunque, alla base di un mutamento epocale: tramite loro nel teatro occidentale la scoperta dell'eros e del suo inveramento scenico e la scoperta conseguente del teatro, specchio formidabile dell'alterità, non solo modificarono e condizionarono lo sviluppo successivo ma incisero anche e soprattutto sull'invenzione del teatro degli attori professionisti, modello fino ad allora mai esperito, e sull'invenzione del pubblico come soggetto collettivo. Così, quando agli occhi di tutti si stagliò la prima costellazione comica femminile, formata da Vincenza Armani, Barbara Flaminia, Lucrezia senese, Angelica Alberghini, Angela Salomone, Vittoria Piissimi, Silvia Roncagli, Isabella Andreini, Diana Ponti, Vittoria Bernardini (per fermarsi ai nomi cronologicamente più alti fin qui emersi dal naufragio delle fonti storiche), si profilò in contemporanea un modello culturale inusitato e sorprendente, che seppe persino andare oltre la recitazione ed affrontare il rischio del capocomicato, misurarsi con l'intelligenza organizzativa e gestionale, campo anche questo fin lì inedito per le donne, fare i conti con la scrittura e con la stampa. Per tali motivi, l'attrice costituì presto un punto di frattura: segnò la cesura tra un prima e un poi e determinò, forse ancor più del nomadismo e della ricerca del mercato, la nascita e l'affermarsi del professionismo.

Il recinto del palcoscenico ${ }^{3}$ le conferì l'esercizio pubblico del potere della finzione, lo sdoppiamento e la moltiplicazione dell'alterità, la possibilità di esercitare il potere intellettivo e seduttivo attraverso il corpo, i gesti, le parole. In un'epoca in cui la disciplina del corpo si faceva serrata e in cui l'ansia prescrittiva rispetto alla fisicità non si fermava nemmeno sulla soglia della camera degli sposi e in cui i discorsi morali sulla corporeità coniugale erano fatti oggetto di dissezione speculativa per entrare persino nei letti, tra le lenzuola di quanti fossero uniti dal

3 Sull'uso del palcoscenico e sul relativo concetto di soglia si veda l'acuto studio di Aliverti, 2011. 
nuovo sacramento del matrimonio ${ }^{4}$, la donna di scena conquistava uno spazio di legittimità espressiva di eccezionale valore storico. Nel suo folgorante apparire, saldava la natura e la cultura: il potere procreativo della donna, misconosciuto dalla coeva teologia, in scena si amplificava, si associava e si saldava al potere creativo. Tra finzione e seduzione, le attrici al loro apparire espressero qualità emotive fin lì sconosciute, assimilate subito in qualità teatrali: la scena fu investita così dall'intensità del sentimento amoroso, materno, combattivo, eroico o doloroso delle donne, il repertorio si dilatò e si infittì di occasioni drammaturgiche, arricchendosi naturalmente in sfumature liriche, tragiche, melanconiche, affettive $^{5}$. Il loro occupare il centro della scena come interpreti ma anche come capocomiche mise in crisi la prescrittiva scientia sexualis, come è stata chiamata da Foucault, che in quel giro di anni si definiva e che avrebbe determinato il discorso sulla sessualità per i secoli futuri, ben oltre l'Ottocento.

Traumatico, a dir poco, fu lo scontro tra il pensiero dominante e la pratica dei comici dell'Arte incardinata sul lavoro delle attrici, tra la Chiesa e il Teatro. A partire dalla seconda metà del Cinquecento, la cultura ufficiale moderna fu fibrillata dalla presenza della sconosciuta tipologia di artista che, a differenza delle pittrici e delle poetesse, delle compositrici e delle stesse cantanti da camera, balzava sulla scena della storia con la sua imbarazzante evidenza pubblica. Soggetto femminile inserito per la prima volta in un contesto artistico collettivo e nomade, l'attrice poteva rinnovare lo statuto identitario delle donne stesse, tanto più che la carica liberatrice della rivoluzione teatrale faceva sì che esse vivessero il clima comunitario che improntava la vita della compagnia nello stesso momento storico controriformato in cui, in modo più forte, si imponevano rigide regole ai comportamenti femminili, in cui si facevano sempre più stringenti le regole della clausura per le donne nei monasteri, ad esempio. E fu questo modello di vita ad essere condannato a viva voce dall'alto dei pulpiti: la convivenza e la coabitazione promi-

4 Il riferimento immediato è ai dieci libri di Tomás Sánchez sul matrimonio, apparsi fra il 1602 e il 1605. Per un'approfondita lettura del trattato si veda Alfieri, 2010.

5 Sulla teoria degli affetti femminili cfr. Ferrone, 2014, pp. 41-42. 
scua avrebbero indotto le donne di sicuro non a commettere «peccato mortale», ma secondo il gesuita spagnolo Pedro Hurtado de Mendoza (Taviani, 1969, p. 88) a esserne la perfetta incarnazione.

Le reazioni suscitate dall'arrivo delle donne in scena, come attrici, cantanti, danzatrici, esecutrici di musica e come capocomiche, non tardarono a manifestarsi. Le testimonianze contro di loro si infittirono, i pareri dei teologi si moltiplicarono a tal punto da far sospettare che fosse luogo comune di tutti i predicatori e di tutta la teologia coeva, cinque-seicentesca, discettare contro quante avessero a che fare con l'arte teatrale: lo dimostra a distanza di cinquant'anni dalla sua uscita il volume di Ferdinando Taviani, La fascinazione del teatro $(1969)^{6}$. In questa silloge, le voci sono tutte concordi nel convocare, in modi e forme più $o$ meno diretti, più o meno velati, il concetto biblico di pietra dello scandalo. Un parametro di giudizio che attraversa le pagine di molti detrattori di parte cattolica post-tridentina: la trattatistica sull'argomento è una grande orchestrazione ripetitiva, una strumentazione retorica che sottende la volontà di formare una coscienza collettiva quale argine contro il fenomeno inarrestabile del teatro professionistico. Un argine che può diventare prevedibile per il lettore avvertito, sebbene la massa delle opinioni in negativo agisca da perfetta cartina di tornasole tramite la quale dimostrare la forza esercitata dalle donne a teatro, come attrici, come capocomiche, come potenziali spettatrici. Basti notare qui come venissero ripresi gli scritti patristici contro le donne e come le auctoritates ricorrenti fossero, oltre Paolo di Tarso, Tertulliano, San Gerolamo, San Giovanni Crisostomo, per limitare la lista ai principali. «Quid est mulier?», "Che cosa (non chi) è la donna?», si chiedeva Tertulliano, e la sua secca domanda è quella che con più insistenza risuonò sulle labbra dei commentatori moderni circa le scandalose donne di scena, l'interrogativo con cui innescare il discorso contro le attrici, siano esse comiche propriamente dette, cantanti, danzatrici o addirittura responsabili di compagnie.

6 Volume inaugurale della collana fondativa degli studi sul fenomeno «La Commedia dell'Arte. Storia Testi Documenti», edita da Bulzoni di Roma e diretta da Ferruccio Marotti. 
Abbacinati dalla bellezza femminile, puntualmente registrata dalle fonti coeve, seppure in negativo, i contemporanei e i posteri hanno sempre insistito sul potere del corpo delle attrici: un potere illimitato, spesso registrato come unico discrimine e spesso da alcune innalzato magistralmente a primo ingrediente del divismo. Nel sollevare la coltre mitopoietica depositatasi intorno alla Commedia dell'Arte, il fenomeno attorico principale per l'Italia, sia per estensione cronologica, sia per diffusione all'estero, si scopre la concordia pressoché unanime dei testimoni intorno alla fascinazione del corpo femminile esibito sul palcoscenico e offerto agli occhi del pubblico, che ha celato e messo in ombra il potere della parola esercitato dalle stesse attrici. Come è noto, il teatro attiva in quanti lo fanno un processo mimetico che pertiene alla fonazione: la messa in voce dell'attrice è funzionale al suo grado di emancipazione tramite la messa in scena. Poiché la mimesi non si risolve con una banale imitazione ma con l'incorporazione di idee ed emozioni, l'esperienza teatrale è tale da cambiare nel profondo, intellettualmente, chi l'attraversa. Il teatro alfabetizza chi lo fa e pertanto alfabetizza le donne, diventa un potente strumento di formazione e di crescita intellettuale e personale, tanto più decisivo in quanto occasione pressoché unica di sviluppo intellettuale per i gruppi sociali ritenuti marginali. È solo il caso di ricordare che sul mondo femminile si abbatté persino il divieto di leggere la Bibbia in volgarizzamenti, interdizione che disorientò quante, laiche e monache, erano state le maggiori fruitrici di questa letteratura (Fragnito, 2003). «Verba muliebris sunt inflammantia», sosteneva San Tommaso, e dunque le attrici a maggior ragione parlano per infiammare senza rimedio chi le ascolta e le rimira, abilmente manipolando l'«artifizio istrionico», come scrisse Cesare Franciotti ne Il giovane Cristiano (1611). Il padre lucchese della Congregazione dei chierici regolari della Madre di Dio ricorreva all'immagine barocca della neve che arde: «Che effetto possiam creder che facciano (le attrici) quando a bello studio, con artifizio istrionico, parlano per infiammare, e di cose poi che da per loro stesse possono far ardere d'impudica fiamma anco la neve?» Ed aggiungeva: «Con le parole si congiungono anco i movimenti della persona, gli sguardi, i sospiri, gli sdegni, e (quel che non può dirsi senza rossore) gli abbracciamenti et altro di peggiore che da queste infernali 
furie in publica scena si vede fare». Nessuno sfugge ai lacci verbali e gestuali delle «impudiche femine», che aumentano a dismisura le loro armi seduttive e arrivano persino a sperimentare il travestitismo di genere, a vestirsi «anco da uomo e saltando e cantando per scena» (Taviani, 1969, p. 178).

La condanna già platonica contro il teatro, contro la poesia, contro gli aedi e dunque contro la tragedia attica, espressa nel X libro della Repubblica, è condanna contro il mezzo di trasmissione del sapere e di educazione del popolo, come scriveva Havelock nel 1963 in Cultura orale e civiltà della scrittura. Il prodigioso modello culturale, messo a fuoco dai primi attori professionisti italiani verso la metà del Cinquecento, fa cadere per sempre l'interdetto che aveva per secoli impedito alle donne la zona della scena, relegandole semmai all'esercizio di arti del teatro di strada o a quello, per sua natura ambiguo e molto praticato durante il Rinascimento, dell'intrattenimento privato principesco sotto forma di poetesse, di musiciste e di cantanti.

Se si analizzano le microstorie delle attrici rinvenute dalla storiografia teatrale ed emerse dalle pieghe della cultura italiana a partire dagli anni Ottanta del Novecento, si nota come le categorie applicate al profilarsi di un teatro che rischiava con le donne l'accesso a inedite pratiche produttive e a inedite funzioni sceniche e che eliminava una volta per tutte il divorzio tra corpo e sesso dalla scena, siano ancorate a giudizi (meglio, pregiudizi) di valore tradizionale. Nel ripercorrere le argomentazioni degli storici della Commedia dell'Arte, a partire da Taviani ${ }^{7}$,

7 Afferma lo studioso: «Si può [...] affermare che da un certo punto in poi le grandi attrici, nel panorama culturale, si trovano al posto delle "meretrices honestae", ne ereditano la cultura e l'arte di tradursi in pubbliche figure. Esse gettano sul mercato vasto della gente disposta a comprare teatro quella stessa cultura, quelle stesse specializzazioni che erano precedentemente riservate a circoli ristretti capaci di mantenersi le proprie etère. In compenso, possono liberare dal commercio la propria persona» (Taviani, in Taviani, Schino, 1982, pp. 336-337, corsivo dell'originale). Poco prima asserisce: «Chi fu, per esempio, quella Lucrezia nominata nel contratto romano del 1564? I suoi sei compagni, il notaio, i testimoni si radunano nella sua casa: una casa in campo Marzio, dove Lucrezia vive come domina, come signora. Ella è l'unica, nel contratto notarile, a comparire con il solo nome e con il luogo d'origine - "domina Lucretia senensis" - mentre di tutti gli altri suoi compagni è detto anche il cognome [...]. 
con poche, significative sfumature si ha l'impressione che si liquidi la questione facendo ricorso alla formula lanciata dal prelato alsaziano Johannes Burckardt, il quale nel Liber Notarum (1498) per le cortigiane rilanciò il titolo di «meretrices honestae», ossia di cortigiane degne di rispetto $^{8}$, riprendendolo da antica tradizione umanistica. La definizione, che fondeva riprovazione e delectatio, corre pressoché intonsa in tutte o quasi le pagine vergate intorno al fenomeno attoriale femminile, come se l'ossimoro utilizzato dal cerimoniere papale fosse di per sé bastante e risolutivo per sbrigare il nodo storiografico, altrimenti spinoso e delicato, riguardante la nuova categoria di donne di scena. Così facendo, è sembrato quasi naturale astrarre il concetto originario riferito alle cortigiane e ricoprire della patina sempreverde del meretricio, seppur d'alto bordo, l'attività artistica e creativa delle attrici e in tal modo svalutarla dalle fondamenta. L'accostamento quando non l'assimilazione tra i due mestieri femminili, l'uno antichissimo per antonomasia, l'altro modernissimo, sono i perni ben oleati sui quali far ruotare le argomentazioni sul professionismo femminile in modo alquanto tendenzioso ${ }^{9}$. Si è così stabilita una coordinata storica di lunga durata e codificata una categoria divenuta durevole, che in modo riflesso e automatico si è applicata al fenomeno ben oltre il periodo aurorale. Il giudizio discriminatorio applicato alle attrici è un'eredità culturale alla quale la storiografia moderna sul professionismo non ha saputo, né voluto rinunciare, manifestando in tal modo di essere contigua allo sguardo obliquo dei primi, acerrimi detrat-

Bastano questi dati a farci intuire qualcosa della sua condizione? Certo no, se cerchiamo dati certi. Sì, invece, se cerchiamo ipotesi plausibili o probabili. La più accettabile, fra queste ipotesi, è allora che la signora Lucrezia appartenga a quel genere particolare di cortigiane che - a Venezia e soprattutto a Roma - eran state famose fin dall'inizio del secolo negli ambienti colti ed aristocratici e che erano dette "meretrices honestae", oneste meretrici, perché facevano professione più di cultura letteraria e di amorosa poesia che di meretricio [...]» (Ibidem, p. 335). Sul motivo delle attrici-meretrici si veda Tessari, 1989. Ancora Ferrone (2014, pp. 52-55) titola un paragrafo alle Attrici 'di piacere', pur disponendo in modo critico i dati su alcune di esse.

8 Fissa il significato di honesta Tamburini, 2016, p. 87n.

9 La derivazione delle attrici dalle meretrices honestae è sviluppata anche da Tamburini, 2016, pp. 85-122, con dovizia di fonti prevalentemente letterarie riguardanti le cortigiane. 
tori delle stesse attrici. Anche negli studi pionieristici sulla Commedia dell'Arte si è data per scontata la propensione moralistica adombrata dai primi detrattori, fonte comoda e rassicurante su cui poggiare l'impalcatura metodologica, sempre difficile e mai raggiunta una volta per tutte, riguardo al fenomeno (Tessari, 2013, p. IX, parla efficacemente di «plesso prismatico», a tal proposito) e su cui concatenare il pensiero e il corrispettivo paesaggio mentale. L'equazione attrice-meretrice, secondo la lingua sciolta di Ottonelli «pestilente pianta di velenosi frutti» (Taviani, 1969, p. 443) ${ }^{10}$, è strisciante anche quando viene sottaciuta, efficiente artificio storico-narrativo da colare con insistita ripetizione, e con una certa qual dose di pruriginosa soddisfazione, sull'immaginario riferito al fenomeno del professionismo femminile.

La storiografia sulle pioniere del teatro professionistico, quando non misconosciuta ${ }^{11}$, sembra avere inizio da un'ambivalenza pregiudiziale. Un'ambivalenza che mescola arte e bisogno, tutela e rischio, redenzione e peccato, nuovo e vecchio, luci e ombre. E che da pregiudizio moralistico scolora presto in stereotipia. Il luogo storiografico divenuto comune dell'attrice-meretrice è il basso continuo ineliminabile e imprescindibile che sottende ogni narrazione che riguardi le donne di scena, una linea di confine assunta come funzionale marcatore storico in grado di segnarne in modo indelebile la presenza sulle tavole dei palcoscenici. Esso consiste in una degradazione del ruolo dell'attrice a lunga gettata storica: molte saranno preoccupate di distanziarsi da questa infamante etichetta (e si pensi, a tal proposito, alle strategie divistiche e nobilitanti dell' «attrice accademica» ${ }^{12}$ Isabella Andreini), molte vi avranno costru-

10 Per alcuni padri della Chiesa, come San Cipriano, l'attrice commette maggior peccato che non la meretrice, perché mentre questa esercita la sua professione in modo privato, quella la esercita in pubblico e dunque incita con «parole e gesti» chi la guardi (Taviani, 1969, p. 138).

11 Colpisce la circostanza che volumi dedicati alla storia delle donne di età moderna contemplino la buona moglie, la donna religiosa, la pittrice ma eclissino ancora l'attrice. Si veda - ad esempio - Calvi, 1992.

12 La definizione è di Meldolesi, 2013, p. 62. Sulla ricca bibliografia dedicata all'attrice, si vedano almeno i contributi raccolti in Guccini, 2004 e Manfio, 2014 e i "classici” Taviani, 1984, Tessari, 1989. 
ito la propria mitopoiesi (e si pensi a Maria Malloni) (Ferrone, 2014, pp. 45, 54), molte altre ancora (le schiere più numerose) l'avranno accettata come una fatalità. L'emancipazione dello sguardo dal dicotomico binario interpretativo (prostituta versus diva, Serva versus Innamorata), induce a ragionare intorno alla costruzione degli aspetti simbolici degli archetipi femminili tradotti in materia teatrale, della loro messa a giorno nel teatro dell'Arte e tentare così un percorso eterodosso intorno alla fenomenologia attorica che più di tutto ne ha segnato lo sviluppo. Se la presenza delle prime donne in scena è tuttora questione storica dirimente, non la si può certo affrontare continuando ad applicare ad essa lo stesso schema di pensiero che l'ha generata, ossia assumendo per paradosso il punto di vista di chi l'ha acerbamente (e altrettanto inutilmente) avversata.

Esercito in movimento e sempre a rischio, sommerso dall'ondata di condanna, le donne attrici, nomadi per mestiere, sono precedute, dunque, da una doppia e simultanea diffidenza: verso le potenzialità precipue dell'arte teatrale professionistica e verso le qualità morali di cui sono state ritenute portatrici non singolarmente, ma come gruppo sociale. Il processo di decostruzione dell'identità artistica delle donne pioniere del teatro non si è limitato al loro primo apparire, bensì ha gettato una lunga e pesante ombra sulle generazioni successive. Non si tratta qui di sostituire al pregiudizio dell'attrice cortigiana l'altra posizione pregiudiziale dell'attrice vergine e santa: piuttosto, si dovrà restituire centralità allo studio dell'attrice, a prescindere dalla sessualità, e dunque trattare le donne di scena alla stessa stregua di come si trattano gli uomini di scena: senza alcun preventivo giudizio moralistico. Il loro arrivo in scena, inquietante per la mentalità dei contemporanei, non può risolversi con una narrazione storica logorata e stancamente stereotipata. Il fissarsi della convezione all'interno di una tradizione storiografica, che vuole le attrici sicure, incontrovertibili e indiscutibili meretrici, può essere sradicata attraverso lo studio accurato delle singole biografie, avvertendo - come scrive Giovanni Pozzi - che la costruzione di un topos coincide perfettamente con la costruzione dell'invenzione della tradizione.

Tenute lontane da sempre dagli strumenti di comunicazione (la pratica della scrittura, quando non è esercizio di aristocratiche, di preferen- 
za è riservata agli ambienti monastici), il teatro ne liberava le potenzialità per la prima volta $\mathrm{e}$ in modo imprevedibile nella storia. Lontane dagli scriptoria, lontane dalle botteghe di pittura o scultura, lontane più che mai dai pulpiti e dagli altari, alle donne in generale, e a quelle dei ceti meno abbienti in particolare, era stato interdetto l'accesso all'espressione. Giova qui ricordare come fosse stato Paolo di Tarso a vietare loro di predicare e di insegnare, con le uniche eccezioni di Tecla (Di Marco, 2007), abbacinata dalla sua predicazione e divenuta prima sua seguace e poi unica apostola, e nel Cinquecento di Teresa d'Avila. Come se questo non bastasse, nelle compagnie dell'Arte non vi era distinzione salariale per genere e per sesso: il lavoro teatrale nella prima età moderna si trasforma in meccanismo di emancipazione femminile straordinario come nessun altro, in veicolo di indipendenza per quante riuscissero a calcare il palcoscenico.

Lo sguardo obliquo, moralizzatore e discriminatorio, che abbassa il vissuto in aneddotica, viene temperato talvolta dalla distinzione manichea tra le oneste e le disoneste, che sottende a sua volta quella tra le sposate e le nubili, e che si estende anche alla stessa arte, se discettano a lungo alcuni stessi comici (ad esempio, Niccolò Barbieri e Pier Maria Cecchini) di teatro onesto e di teatro disonesto. A distanza di secoli dai pronunciamenti dei primi censori, alle attrici si applica una rigida schematizzazione: da una parte, le accompagnate, e dunque per questo meritevoli dell'attributo dell'onestà, dall'altra, le scompagnate, e dunque per questo rifluite tra quelle che tra le oneste propriamente dette non possono rubricarsi. La differenza formale e giuridica, in questa insistita gerarchia còlta nel suo grado zero, la fa il matrimonio. E ciò non è un caso fortuito, né banale. Corre l'obbligo di ricordare che il sacramento del matrimonio e il relativo vincolo furono confermati per mezzo del decreto Tametsi nel 1563, emanato dal Concilio tridentino, che chiuse i battenti in prossimità cronologica con l'arrivo di alcune celebri donne in scena: la romana Barbara Flaminia e la senese Lucrezia, balzate sui palcoscenici dell'Arte proprio in quel torno di anni, rispettivamente nel 1562 e nel 1564. Seppure i comici professionisti, seguendo il comportamento di ampi strati della popolazione, sulle prime in larga parte seppero sfuggire alle imposizioni 
controriformate in materia di unione coniugale e continuarono nella pratica consuetudinaria del concubinato, è certo che le uniche attrici di cui abbiamo notizia sono quelle che hanno agito dietro lo scudo della tutela maritale. A partire da Angelica Alberghini, moglie di Drusiano Martinelli, da Barbara Flaminia, moglie di Alberto Naselli, dalla più nota Isabella, universalmente conosciuta come Andreini che come Canali, ad Orsola Posmoni sposata con Pier Maria Cecchini, alle due Virginie (Rotari e Ramponi) vissute nel cono d'ombra e nel gioco teatrale dell'Innamorato Lelio-Giovan Battista Andreini, a Salomé Antonazzoni sposata con Giovan Battista Austoni, alle drammaturghetraduttrici Brigida Bianchi e Orsola Cortesi, la prima impalmata da tre attori, la seconda compagna dell'Arlecchino Domenico Biancolelli fino a Maddalena Facchinetti, moglie ad intermittenza del Brighella Giuseppe Marliani, l'attrice che ispirò alcune, determinanti opere della riforma goldoniana (Bonomi-Vescovo, 2018).

Congiunta dal sacramento del matrimonio normato dal Concilio di Trento o unione di fatto, la coppia è la cellula del teatro all'improvviso, intorno alla quale si strutturano geneticamente e artisticamente le famiglie dell'Arte. L'unione si rifrange nella struttura produttiva e organizzativa delle compagnie (come, per riflesso, nello scioglimento degli intrecci comici), pur avvertendo che le sacramentate siano quelle meglio viste e più favorite nella considerazione della cultura controriformata. La coppia, sposata o meno, nel legittimare la presenza delle donne in scena, rompe anche l'isolazionismo sociale al quale soprattutto le appartenenti, numerose, ai ceti meno abbienti erano di sicuro condannate. Le relazioni affettive si tramutano in relazioni teatrali e sono il viatico che consente alle donne di entrare nell'agone scenico, di sottrarsi alla taccia di immoralità sempre sottesa in ogni loro azione pubblica con la creazione dello spazio comunitario protettivo e difensivo costituito dalla compagnia: la microsocietà degli attori, chiusa e necessariamente autoreferenziale, messa a fuoco per tempo da Claudio Meldolesi (2013, pp. 57-77). Certo è che, creato il problema identitario intorno alle pioniere del teatro, innestato il virus della disonestà in ogni piega che le riguardasse, diffuso in ogni dove e in ogni come l'odore salmastro di condotte licenziose, ribadito lo spavento del loro corpo dalle pagine teologiche autoritative, 
non si possa del tutto escludere che qualcuna abbia negato se stessa pur di essere accettata.

L'accusa di disonestà lanciata contro le attrici produceva effetti di instabilità gravi, capaci di disgregare la vita delle neonate compagnie. Ma cosa intaccava realmente tale accusa? L'invenzione della disonestà delle attrici, costruita sul tavolo della teologia anche con l'attrezzo ideologico, duro a morire, della misoginia, si riverberava nel pensiero contemporaneo e successivo, tanto da istituire una tradizione, a cui sfuggono solo attrici anomale, eccezioni - si sa - che confermano la regola generale. Il meretricio steso su di loro, con le poche eccezioni dei nomi tenuti a galla dalla storiografia, intaccava in modo indelebile il concetto di onore, cardine della società maschile europea sinonimico dell'ordine patriarcale. Occorre riflettere e ripensare su come il codice dell'onore sia stato l'asse portante del potere delle élite nobiliari di antico regime e su come sia stato causa scatenante all'origine dell'iterato tentativo di delegittimare 1'arte rappresentativa femminile. José Antonio Maravall ha dimostrato nei dettagli quanto l'onore, concetto solidale all'autorità, sia stato fondamentale nella costituzione della società assolutistica europea. Ha scritto lo studioso: «la difesa della mascolinità rientra nel programma integrativo della società che spetta all'onore», chi «difende la reputazione secondo la concezione maschile vigente della moglie, della sorella, della figlia, libera dunque la società da un pericolo disgregante» (1984, p. 72).

Quante furono le attrici dell'Arte? L'interrogativo non è banalmente quantitativo. Al netto di lacune storiografiche ancora non quantificabili, a giudicare dagli echi che seppero provocare e dallo scandalo prodotto sembrerebbero moltissime. In realtà, la loro presenza nelle compagnie dell'Arte fu dissimile a seconda dell'orizzonte geografico e culturale dal quale guardiamo al fenomeno. Mentre sono assestate nelle compagnie italiane del circuito del Centro-Nord, altrettanto non può dirsi per Roma e per il circuito meridionale. Mentre le cosiddette compagnie lombarde e quelle del centro Italia, legate alle corti ducali e granducali di Mantova, di Ferrara, di Firenze, prevedono le donne, a Roma e a Napoli vigevano altri criteri. A Roma l'interdizione contro le attrici è radicata almeno quanto quella contro i comici, seppure le eccezioni - consentite 
quasi esclusivamente nel periodo del carnevale - vengono determinate volta volta dalla politica dei papi. Altrove ho dimostrato il ruolo pressoché residuale svolto dalle donne artiste nelle compagini dei professionisti agenti a Napoli (Megale 2017, pp. 63-71). Nella capitale vicereale le attrici sembrano non occupare lo spazio artistico che invece avevano saldamente conquistato nei circuiti teatrali del Nord e del Centro Italia. Sui teatri pubblici partenopei agivano di preferenza dive "lombarde" e dive "spagnole", non dive indigene. Colpisce la circostanza della reticenza delle fonti storiche finora emerse rispetto alla presenza/assenza delle attrici: un'intermittenza forse foriera di una generale instabilità artistica, di un bisogno non impellente di presentare personaggi sessuati, risolto diversamente sotto il Vesuvio, con il ricorso convenzionale ad attori maschi per incarnare il genere femminile. Più delle napoletane propriamente dette, rare se non del tutto assenti, vere e proprie convitate di pietra di quel teatro, nelle compagnie attive in città, agivano di preferenza le spagnole e, in taluni casi, attrici dell'hinterland, come la nolana Margarita Candida, seppure tutt'altro discorso potrebbe farsi circa le attrici presenti nelle troupe che percorrevano in lungo e in largo le numerose province del Regno di Napoli.

Riguardo alle tecniche recitative, occorre rilevare come la maschera, componente strutturale del linguaggio scenico della Commedia dell'Arte, non riguardasse la donna attrice, neanche quando al posto dell'Innamorata, parte dal volto scoperto anche per gli attori, si profilasse la Serva, parte invece sempre mascherata quando è sostenuta dagli attori. L'attrice è senza maschera anche quando assolve a parti ritenute non fisse, ma mobili: quando interviene sul palcoscenico alla bisogna come Ruffiana, Maga, Ostessa, Nutrice, Astrologa. La forma per eccellenza del teatro comico, la mezza maschera che più di ogni altro elemento denota lo stile recitativo all'improvviso, non appartiene alle donne, impiegate semmai in esibizioni sensuose quando non in numeri da strip tease (Tessari, 2013, p. X), ma sempre a volto nudo. La bellezza femminile doveva essere esposta nella sua integrità e non velata: il volto scoperto doveva essere epitome del corpo e veicolo di ben altre nudità, l'attrazione scenica resa in tutta la sua carica di seduzione. La donna svolge sul piano recitativo un ruolo fondamentale nella proposizione 
e nella credibilità di maschere e lazzi e dei tanti generi (farsa, tragedia, pastorale, tragicommedia) allestiti dai comici. Talvolta, ed è il caso arcinoto di Isabella Andreini, può svolgere anche il ruolo di tramite tra la cultura alta e quella bassa, tra le pratiche sceniche del teatro di corte e quelle dei teatro degli istrioni. Poi, con il suo arrivo sui palcoscenici si scatenano nuove dinamiche relazionali. Come in uno scatto rubato dal backstage, fu al solito un gesuita a cogliere la complicità artistica tra attrici e attori: «l'uomo spoglia la donna e la veste, perché ella senza perdere tempo possa assumere nella commedia ruoli diversi. (...) il giovane allaccia le scarpette della donna e lega i calzari alti con striscioline di seta, non solo sulla scena, ma anche vicino al letto» ${ }^{13}$. L'attore che aiuta la donna a travestirsi e a legarsi le scarpette, è un uomo, che si consegna però, nelle sue mani: l'antropologia ha spiegato il valore sessuale delle scarpe, a partire da quelle favolose della Gatta Cenerentola di Giovan Battista Basile. Come i comici con le tecniche dell'improvviso esularono dall'inventio e dalla compositio del testo drammatico, così le donne furono le complici attive di una praxis rinnovata dello spettacolo.

Sia le attrici dive (prevalentemente Innamorate), sia le attrici prostitute (prevalentemente Servette), che quelle penitenti e convertite in controriformate pose maddalenesche o addirittura in pose egiziache - le categorie nelle quali fin qui sono state sistematizzate le prime attestazioni del professionismo femminile - furono attraversate dalla medesima angoscia della violenza, dato sociale che oltrepassa di gran lunga l'età moderna e che tuttavia trovò un canale preferenziale sul corpo scenico delle donne attrici. E sul quale occorre riflettere per comprendere meglio come soprusi e angherie avessero inciso sul cammino artistico e sul vissuto delle interpreti pioniere dell'Arte, e come le avessero condizionate, l'ombra di Artemisia Gentileschi propagandosi su tutte. Fra maschere d'autore e pregiudizi storiografici circa la perturbante percezione estetica rappresentata dall'ingresso delle donne in scena, decostruire ciò che separa il mestiere di attrice dalle vicende sessuali, il talento dalla re-

13 Hurtado de Mendoza, Scolasticae et morales disputationes, 1631 in Taviani, 1969, pp. 87-88. 
putazione, è essenziale per dare nuove categorie e nuove coordinate al complesso problema e per superare certa tendenza al biografismo.

I casi di attrici picchiate, quando non anche violentate, non dovettero essere né rari, né pochi. Anzi. A giudicare dalla fine per avvelenamento nel 1569 riservata ad una delle primissime professioniste note, Vincenza Armani, e da quella, misteriosa, di Caterina Martinelli nel 1608 , la morte violenta poteva concludere addirittura la loro stessa professione. La difficoltà nel reperire documenti in tal senso conferma, una volta di più, la lettura foucaultiana circa l'«infamità» di vite, «destinate a passare al di sotto di qualunque discorso e a sparire senza mai essere state dette (...) se non nel punto del loro istantaneo contatto con il potere» (Foucault, 2009, pp. 22-23). Sfrondate dalle menzogne e dalle parzialità interpretative in cui sono imprigionate le testimonianze residue, sappiamo in controluce del tentativo di sfregio indirizzato al volto di Angelica Alberghini nel 1591; della moglie del comico Francesco Galta, dal nome sconosciuto, che sfuggì a uno stupro di gruppo nel 1633, mentre compiva un corso di recite tra Crotone e Catanzaro, in Calabria (Megale, 2017, pp. 85, 381-382); di Bernarda Ramírez che nel 1637 fu rapita da Jerónimo López, duca di San Pietro; di Isabel de Andriago, abusata da un cavaliere di Siviglia; di María de León, rapita dall'almirante di Castilla qualche giorno prima delle nozze con l'attore Alonso de Olmedo; di Isabel de Gálvez, nel novembre del 1657 prelevata direttamente dalle tavole del palcoscenico e sequestrata a Madrid dal conte di Monterrey e dal marchese de Almazán. A questi citati, si aggiunga l'episodio storico riguardante la biografia dell'attrice Antonia de Ribera, prima donna della compagnia di Roque de Figueroa, attiva a Napoli almeno negli anni trenta del Seicento. La nota fuga a Livorno della de Ribera con il principe Pompeo Colonna, che mise a repentaglio le relazioni fra alcune famiglie apicali della prima metà del XVII secolo (i Colonna, i d'Avalos, i Medici), imbarazzò la diplomazia di alcuni Stati (quella vicereale e quella granducale) e tenne in fibrillazione per qualche mese i rapporti politici fra la Spagna e la Toscana, non cela nulla di romantico, né di romanzesco. Acquista, semmai, valore paradigmatico della violenza (fisica, oltre che psichica) subita dalle donne di spettacolo e, insieme, della persistenza di pregiudizi storiografici con i quali si 
continua a guardare al mestiere di attrice. Il "caso" della de Ribera che, per sfuggire alle violenze del viceré Monterrey, rinunciò al palcoscenico e scelse la monacazione, è un episodio esemplare della necessità di guardare ai comportamenti delle attrici dell'Arte con sguardi non viziati da stereotipi, né da preconcette letture a lungo stratificatesi intorno alla costruzione degli aspetti simbolici degli archetipi femminili ${ }^{14}$.

Persino l'iniziazione al mestiere di attrice, quando non si aveva la fortuna di appartenere a una famiglia d'Arte, garante di una trasmissione regolare e "naturale", poteva essere marchiata da aggressività e da prepotenze di ogni tipo ${ }^{15}$ : inevitabile fio da pagare in cambio dell'acquisizione dei saperi teatrali. L'elenco appena accennato dei crimini nascosti, fin troppo parziale e limitato quasi esclusivamente alle interpreti spagnole, dà pienamente ragione a chi, come Virginia Rotari, in arte Lidia, nel 1628 «madre vedova e carica di sette figliuoli», considerava il mestiere di attrice «esercizio tanto pericoloso per donna» ${ }^{16}$ da cercare di evitarlo ad almeno una delle sue figlie. Infinito poi sarebbe il gioco delle corrispondenze fra le biografie e la drammaturgia. Basti qui accennare a come le scene delittuose sublimate nelle pastorali, negli incontri tra i satiri e le ninfe, o in tutte le guerre d'amore allestite a teatro, siano la trasfigurazione di meccanismi violenti, camuffati dal riverbero della finzione. Anche in tal caso, connotare i comportamenti delle attrici di soli significati sessuali è il modo attraverso il quale abbassare il tono della discussione critica fino al punto da renderla superflua ed evanescente, legandola al solo narcisismo dei protagonisti maschili, quelli effettivi delle trame storiche e quelli che ne interpretano, ne leggono e ne

14 L'esemplare vicenda, comprovata dalla rilettura di documenti d'archivio fin qui letti in modo erroneo o parziale, è ricostruita in Megale, 2016, pp. 103-128. La violenza esercitata sulle donne, fenomeno antichissimo e di vastissima portata, è indagata per il Medioevo da Esposito, Franceschi, Piccinni, 2018.

15 È quanto - ad esempio - lascia trapelare l'episodio di una bambina napoletana affidata al capocomico spagnolo Sancho de Paz perché la avviasse alla carriera di attrice (Megale, 2017, pp. 34-35).

16 Lettera autografa di Giovan Battista Andreini per conto di Virginia Rotari a Maria Gonzaga, Vienna, 16 novembre 1628, Archivio di Stato di Mantova, Gonzaga, b. 495, lettera 7, in Ferrone, et al., 1993, I, pp. 145-146. 
fruiscono a posteriori. Dietro le quinte e sui palcoscenici la violenza di genere attua la morale, cristiana prima e borghese poi, della negazione e del disconoscimento della sessualità. Nella prospettiva foucaultiana del bio-potere, il disconoscimento del corpo dell'attrice è la conferma stessa della forza delle istituzioni. Tra aporie e anomalie, tra scarti ed eccezioni, tra norma e trasgressioni la cultura delle attrici ha condizionato, determinato e governato la cultura teatrale. E spesso l'ha generata, addirittura procreata.

\section{BIBLIOGRAFIA}

Alfieri, F. (2010). Nella camera degli sposi. Tomás Sánchez, il matrimonio, la sessualità (secoli XVI-XVII). Bologna: il Mulino.

Aliverti, M.I. (2011). Problematiche di soglia e problematiche di genere nella Commedia dell'Arte tra fine Cinquecento e inizio Seicento: un'ipotesi di lavoro. In G. Di Palma, L. Mariti, L. Tinti \& V. Valentini (Eds.), Studi e testimonianze in onore di Ferruccio Marotti. III. Lo spettacolo dall'Umanesimo al Barocco. La Commedia dell'Arte (pp. 197-227). Roma: Bulzoni.

Bonomi, S., \& Vescovo, P. (2018), «In due si fanno l'opre famose»: il sodalizio Goldoni-Medebach. Studi goldoniani, 7, 45-86.

Calvi, G. (Ed.). (1992). Barocco al femminile. Roma-Bari: Laterza.

Di Marco, F. (2007). Tecla di Icono e le donne alla finestra. Storia delle donne, 3, 77-98.

Esposito, A., Franceschi, F., \& Piccinni, G. (Eds.). (2018). Violenza alle donne. Una prospettiva medievale. Bologna: il Mulino.

Ferrone, S., Burattelli, C., Landolfi, D., \& Zinanni, A. (Eds). (1993). Comici dell'Arte. (2 voll). Firenze: Le Lettere.

Ferrone, S. (1993). Attori mercanti corsari. La Commedia dell'Arte in Europa tra Cinque e Seicento. Torino: Einaudi.

Ferrone, S. (2014). La Commedia dell'Arte. Attrici e attori italiani in Europa (XVI-XVIII secolo). Torino: Einaudi.

Foucault, M. (2009). La vita degli uomini infami. Bologna: il Mulino.

Fragnito, G. (2003). Censura ecclesiastica e identità spirituale e culturale femminile. Mélanges de l'école française de Rome, 115-1, 287-313. 
Guccini, G. (2002-2003). Intorno alla prima 'Pazzia d'Isabella'. Fonti-intersezioni-tecniche. Culture teatrali, 7/8, 167-207.

Guccini, G. (Ed.). (2004). L'arte dei comici. Omaggio a Isabella Andreini nel quarto centenario della morte (1604-2004). Culture teatrali, 10 (numero monografico).

Inventione di Giulio Pallavicino di scriver tutte le cose accadute alli tempi suoi: 1583-1589, a cura di E. Grendi. (1975). Genova: Sagep.

MacNeil, A. (2003). Music and Women of the Commedia dell'Arte in the Late Sixteenth Century. New York: Oxford University Press.

Maiorana, B. (1996). Finzioni, imitazioni, azioni: donne e teatro. In G. Zarri (Eds.), Donna, disciplina, creanza cristiana dal XV al XVII secolo (pp. 121-139). Roma: Edizione di Storia e Letteratura.

Manfio, C. (Ed.). (2014). Isabella Andreini una letterata in scena. Padova: Il Poligrafo.

Maravall, J.A. (1984). Potere, onore, élites nella Spagna del secolo d'oro. Bologna: il Mulino.

Marotti, F., \& Romei, G. (1991). La Commedia dell'Arte e la società barocca. La professione del teatro. Roma: Bulzoni.

Mattioda, E. (2014), Giorgio Vasari, l'attrice Flaminia romana e Leone de' Sommi. In G. Baldassarri, V. Di Iasio, P. Pecci, E. Pietrobon \& F. Tomasi, La letteratura degli italiani 4. I letterati e la scena. Atti del XVI Congresso Nazionale Adi (Sassari-Alghero, 19-22 settembre 2012) (pp. 481-522). Roma: Adi.

Megale, T. (2016). Antonia de Ribera dal palcoscenico al chiostro. In fuga dalla violenza maschile. Drammaturgia, XIII, n.s. 3, 103-128.

Megale, T. (2017). Tra mare e terra. Commedia dell'Arte nella Napoli spagnola (1575-1656). Roma: Bulzoni.

Meldolesi, C. (2013). Pensare l'attore, ed. L. Mariani, M. Schino \& F. Taviani. Roma: Bulzoni.

Sánchez, T. (1602,1605). Disputationes de sancto matrimonii sacramento. Genova: Pavoni.

Simoncini, F. (2016). Barbara Flaminia attrice e cantante tra piazza, corte e accademia. Ipotesi di collaborazione con Giorgio Vasari e Bernardo e Torquato Tasso. In S. Brunetti (Ed.), Maestranze, artisti e apparatori per la scena dei Gonzaga (1480-1630). Atti del Convegno Internazionale di studi (Mantova, 26-28 febbraio 2015) (pp. 304-315). Bari: Edizioni di Pagina. 
Tamburini, E. (2016). Culture ermetiche e Commedia dell'arte. Tra Giulio Camillo e Flaminio Scala. Ariccia (Roma): Aracne.

Taviani, F. (1969). La Commedia dell'Arte e la società barocca. La fascinazione del teatro. Roma: Bulzoni.

Taviani, F. (1984). Bella d'Asia. Torquato Tasso, gli attori e l'immortalità. Paragone Letteratura, XXXV, 408-410, 3-76.

Tessari, R. (1989). O Diva, o «Estable à tous chevaux». L'ultimo viaggio di Isabella Andreini. In Viaggi teatrali dall'Italia a Parigi fra Cinque e Seicento. Atti del Convegno Internazionale (Torino, 6-8 aprile 1987) (pp. 128-142). Genova: Costa \& Nolan.

Tessari, R. (2013). La Commedia dell'Arte: genesi d'una società dello spettacolo. Roma-Bari: Laterza.

Riassunto: Nella seconda metà del Cinquecento, l'arrivo delle donne in scena provocò una rivoluzione i cui effetti si estesero all'intera società moderna. Sommerse da un'ondata di condanna, le attrici, nomadi per mestiere, furono precedute da una doppia e simultanea diffidenza: verso le potenzialità precipue dell'arte teatrale professionistica e verso le qualità morali di cui sono state ritenute portatrici non singolarmente, ma come gruppo sociale. Il processo di decostruzione dell'identità artistica delle attrici pioniere, assimilate alle meretrici o al loro rovescio, ossia all'attrice vergine, non si è limitato al primo apparire del professionismo femminile, bensì è diventato una categoria storica di lunga durata. Si tratta qui di rimuovere i preventivi giudizi moralistici stratificati sulle attrici e sgombrare il campo storiografico da posizioni preconcette, di smantellare misoginia e anacronistici sguardi obliqui. Fra maschere e pregiudizi circa la perturbante percezione estetica rappresentata dall'ingresso in arte della donna, distinguere ciò che separa il mestiere di attrice dalle vicende sessuali, il talento dalla reputazione, l'arte dalla seduzione è essenziale per dare nuove categorie storiografiche e nuove coordinate allo studio della Commedia dell'Arte. Vengono così alla luce altri vissuti e altre esperienze: l'avvio verso la professione, il ruolo del matrimonio, la violenza subita sono alcuni nodi problematici da riconsiderare e vagliare sulla scorta di preventive indagini documentarie.

Parole chiave: professionismo femminile, capocomicato femminile, drammaturgia delle donne, Commedia dell'Arte, storiografia teatrale 